\title{
生态系统结构与功能：前沿与展望
}

\author{
王志恒 ${ }^{1^{*}}$ 刘玲莉 ${ }^{2 *}$
}

${ }^{1}$ 北京大学城市与环境学院生态研究中心, 地表过程分析与模拟教育部重点实验室, 北京 $100871 ;{ }^{2}$ 中国科学院植物研究所植被与环境变化国家重点实 验室, 北京 100093

王志恒, 刘玲莉 (2021). 生态系统结构与功能: 前沿与展望. 植物生态学报, 45, 1033-1035. DOI: 10.17521/cjpe.2021.0370

\section{Ecosystem structure and functioning: current knowledge and perspectives}

\author{
WANG Zhi-Heng ${ }^{1 *}$ and LIU Ling-Li ${ }^{2 *}$
}

${ }^{1}$ Institute of Ecology, College of Urban and Environmental Sciences and Key Laboratory for Earth Surface Processes of the Ministry of Education, Peking University, Beijing 100871, China; and ${ }^{2}$ State Key Laboratory of Vegetation and Environmental Change, Institute of Botany, Chinese Academy of Sciences, Beijing 100093, China

Wang ZH, Liu LL (2021). Ecosystem structure and functioning: current knowledge and perspectives. Chinese Journal of Plant Ecology, 45, 1033-1035. DOI: 10.17521/cjpe.2021.0370

生态系统是由生物群落及其生存环境共同组 成的动态平衡体系, 是生态学研究的基本单元。自 20世纪30年代提出生态系统概念(Tansley, 1935)以 来, 生态系统生态学逐渐成为生态学研究的核心方 向之一, 形成了完备的理论与方法体系。近年来, 全 球变化不断加剧, 并已对生态系统的结构和过程产 生了深远的影响。解析生态系统组成、结构及其动 态, 阐明其对生态系统功能和服务的影响, 是认识 生态系统如何响应全球变化的基础, 因此越来越受 到生态学家的关注。

在中国科学院生命科学与医学学部和地学部 的联合资助下, 方精云院士负责的“生态系统生态 学学科发展战略咨询项目”于2016年启动。在该项目 的资助下, 我们组织了《生态系统结构与功能》专 辑, 共收录了9篇文章, 分别就生态系统营养级互 作、食物网和生态网络及对全球变化响应的前沿进 展进行了系统梳理。

生态系统中生物群落由生产者、消费者和分解 者构成, 三者之间通过取食和被取食过程, 形成营 养层级结构和网络关系, 驱动生态系统的物质循环 和能量流动。Elton (1927)在其经典著作《动物生态 学》中最早提出了食物网(food web)这一概念, 指出 群落中的各条食物链相互关联, 构成食物网。在 Elton工作的基础上, Lindeman (1942)提出了营养级 动态(trophic dynamic)的概念和著名的“十分之一定 律”: 即能量在金字塔型的食物链内逐级流动时, 传 递效率约10\%。从20世纪70年代开始, May (1972)关 于生态网络复杂性与稳定性的研究将食物网的研究 拓展到生态网络, 极大促进了生态系统生态学的研 究。生态系统的营养结构、食物网和生态网络研究 是理解生态系统的组成、结构与动态的理论基础, 并为生物多样性保护、生态系统管理与恢复以及应 对全球变化等方面提供了不可或缺的科学支持, 因 而成为当前生态系统生态学的前沿方向之一。

不同营养级的互作机制是食物网理论的基础, 而其中植物与植食性动物的相互作用是最重要的一 种种间关系。钟志伟等(2021)综述了植食性动物对 植物个体、种群和群落的影响, 总结了植物在长期 进化过程中为防御植食性动物形成的一系列化学和 物理防御策略, 并对相关重要假说进行了总结和比 较。不同物种间的相互作用形成了复杂的生态网络, 这些相互作用包括拮抗、互惠、竞争、偏害共栖以 及共生等。李海东等(2021)综述了关于种间互作网 络的结构特征、构建机制、稳定性机制等方面的研 究进展, 并系统探讨了种间互作网络在解析生态系 统结构多样性与生态系统功能维持机制方面的推动 作用。

人类活动改变了生态系统的营养结构和食物

收稿日期Received: 2021-10-13 接受日期Accepted: 2021-10-15

基金项目: 国家自然科学基金委员会基础科学中心项目(31988102)。Supported by the National Natural Science Foundation of China for Basic Science Center Project (31988102).

* 同等贡献Contributed equally to this work (Wang ZH, zhiheng.wang@pku.edu.cn; Liu LL, lingli.liu@ibcas.ac.cn) 
网组成, 从而极大地扰动了生态系统的物质循环和 能量流动。王晴晴等(2021)聚焦于气候变化、过度 捕猎、栖息地丧失等导致的营养级错位、关键种丧 失和生物入侵加剧等过程, 综述了全球变化下食物 网结构改变的机制。如何准确预测环境变化下生态 系统组成与结构的时空动态是防止生态系统退化、 提升生态系统适应性管理的科学基础。贺强(2021) 在概述连续型、阈值型和随机型等生态系统动态模 式的基础上, 对生物互作如何影响生态系统动态的 主要进展进行了总结, 并探讨了生物互作理论在生 态系统保护和修复中的应用。

生物多样性是支撑人类生存与社会可持续发 展的基本自然资源。狭义的生物多样性通常指特定 时空内所有生命体的多样性或变异; 广义的生物多 样性则指生物与环境形成的生态复合体, 以及与此 相关的各种生态过程的总和(Wilson, 1988; 马克平, 1993; Hooper et al., 2005)。生物多样性是组成生态 系统的基本要素, 而生态系统则是生物多样性的载 体。生物多样性包含多个层次和维度, 包括遗传多 样性、物种多样性、功能多样性、系统发育多样性 以及生物与环境耦合所形成的群落、生态系统和景 观的多样性。生物多样性是生态系统功能和服务形 成的基础, 可对生产力、物质循环等生态系统功能 和服务及其稳定性产生显著影响。随着人类对环境 的干扰越来越强, 生物多样性正在面临快速丧失的 风险, 生物多样性危机及其生态系统后果逐渐显 现。因此, 近年来, 生物多样性在维持生态系统功能 和服务方面的作用越来越为人们所重视。这使得有 关生物多样性与生态系统功能关系(BEF)的研究逐 渐成为当前生态系统生态学中最活跃的一个方向。

近年来, 有关生物多样性与生态系统功能及 其稳定性的研究取得了长足进步。为检验生物多样 性对生态系统功能及其稳定性的影响, 生态学家在 全球不同地区开展了数百个室内外生物多样性实验

(Hooper et al., 2012; Tilman et al., 2014; Isbell et al., 2015)。研究显示, 生物多样性与生态系统功能及其 稳定性有极为复杂的联系。为解释生物多样性对生 态系统功能和服务及其稳定性的影响, 生态学家提 出了多种理论。在这一领域, 近年来最引人注目的 进展包括生物多样性对生态系统多功能性和多服务 性的影响以及生态系统的多尺度稳定性框架等。井 新和贺金生(2021)在回顾生物多样性与生态系统多
功能性和多服务性关系研究历史的基础上，系统梳 理了这一问题的主要研究进展和发展趋势, 特别关 注了生物多样性与生态系统多功能性关系研究的新 方法; 而张宏锦和王娓(2021)则关注土地利用变化、 气温升高、降水改变以及氮沉降等全球变化因子对 生态系统多功能性的影响; 同时, 两组作者均提出: 在未来研究中, 应规范生态系统多功能性的评估方 法并重点关注全球变化对生物多样性与生态系统多 功能性关系的影响。与这两组作者不同, 李周园等 (2021)则聚焦生物多样性对生态系统稳定性的影 响。他们首先梳理了近期关于生态系统稳定性内涵 的新认识以及多样性-稳定性关系研究进展, 并详 细综述了最近发展起来的多尺度稳定性理论框架, 提出了本领域的未来发展建议。

功能性状指能影响有机体适合度的形态、生理 或物候性状, 反映了生物对生存环境的适应策略 (Violle et al., 2007)。近年来, 人们在不同地区, 对不 同类群的功能性状开展了大量研究, 功能性状数据 不断积累, 这极大促进了人们对功能性状重要性的 认识。植物功能性状可在多个尺度影响生态系统过 程与功能。在个体和种群水平, 功能性状及其权衡 可影响有机体生长、存活和繁殖等生存策略; 在群 落和生态系统尺度, 功能性状可影响物种共存和群 落构建。同时, 由于具有不同功能性状的物种对环 境变化的响应不同, 功能性状也可影响生态系统功 能和服务及其对全球变化的响应。有关功能性状(特 别是植物功能性状)的时空分布和成因及其对生态 系统结构与功能的影响日益为人们所重视。

潘权等(2021)系统综述了植物功能性状对生 态系统服务的影响及其机制, 阐明了多个植物性状 对生态系统服务的影响途径; 而李耀琪和王志恒 (2021)则系统综述了植物叶片形态特征的时空格局 及其驱动因素, 讨论了叶片形态及其权衡对生态系 统功能的影响及其在模拟或重建生态系统功能中的 应用。在此基础上两组作者提出, 未来研究应聚焦 植物功能性状相关性、气候变化和人类活动、时空 尺度差异等因素对植物功能性状及其与生态系统服 务关系的影响, 并利用多种新技术进一步增加植物 功能性状数据的积累。

生态系统的结构与功能紧密相关, 是理解生态 系统动态变化及其对全球变化响应的关键。本专辑 综述了有关生态系统结构和功能研究的最新进展, 
所涉方向均为近年来的热点领域, 以期抛砖引玉。 未来的研究需结合新的观测技术、实验手段和理论 模型, 进一步探讨生物多样性与生态系统功能和服 务的关系及其对全球变化的响应; 揭示典型生态系 统营养结构和生态网络结构及调控机制, 最终整合 食物网、生态网络、生物多样性、生物地球化学循 环等理论, 深化人们对生物多样性丧失可能带来的 系统性后果的认识, 为生态系统管理和保护提供科 学指导, 也为生态系统多功能性的维持、应对气候 变化等国家重大需求提供理论支持。

致谢 此专辑由中国科学院学部学科战略发展研究 项目“生态系统生态学” (2016-SM-B-01)及国家自然 科学基金委员会基础科学中心项目(31988102)资助。

\section{参考文献}

Elton CS (1927). Animal Ecology. Sidgwick \& Jackson, London. He Q (2021). Biotic interactions and ecosystem dynamics under global change: from theory to application. Chinese Journal of Plant Ecology, 45, 1075-1093. [贺强 (2021). 生物互 作与全球变化下的生态系统动态: 从理论到应用. 植物 生态学报, 45, 1075-1093.]

Hooper DU, Adair EC, Cardinale BJ, Byrnes JEK, Hungate BA, Matulich KL, Gonzalez A, Duffy JE, Gamfeldt L, O’Connor MI (2012). A global synthesis reveals biodiversity loss as a major driver of ecosystem change. Nature, 486, 105-108.

Hooper DU, Chapin FS, Ewel JJ, Hector A, Inchausti P, Lavorel S, Lawton JH, Lodge DM, Loreau M, Naeem S, Schmid B, Setala H, Symstad AJ, Vandermeer J, Wardle DA (2005). Effects of biodiversity on ecosystem functioning: a consensus of current knowledge. Ecological Monographs, 75, 3-35.

Isbell F, Craven D, Connolly J, Loreau M, Schmid B, Beierkuhnlein C, Bezemer TM, Bonin C, Bruelheide $\mathrm{H}$, de Luca E, Ebeling A, Griffin JN, Guo Q, Hautier Y, Hector A, et al. (2015). Biodiversity increases the resistance of ecosystem productivity to climate extremes. Nature, 526, 574-577.

Jing X, He JS (2021). Relationship between biodiversity, ecosystem multifunctionality and multiserviceability: literature overview and research advances. Chinese Journal of Plant Ecology, 45, 1094-1111. [井新, 贺金生 (2021). 生 物多样性与生态系统多功能性和多服务性的关系: 回 顾与展望. 植物生态学报, 45, 1094-1111.]

Li HD, Wu XW, Xiao ZS (2021). Assembly, ecosystem functions, and stability in species interaction networks. Chinese Journal of Plant Ecology, 45, 1049-1063. [李海东, 吴新 卫, 肖治术 (2021). 种间互作网络的结构、生态系统功
能及稳定性机制研究. 植物生态学报, 45, 1049-1063.]

Li YQ, Wang ZH (2021). Leaf morphological traits: ecological function, geographic distribution and drivers. Chinese Journal of Plant Ecology, 45, 1154-1172. [李耀琪, 王志 恒 (2021). 植物叶片形态的生态功能、地理分布与成因. 植物生态学报, 45, 1154-1172.]

Li ZY, Ye XZ, Wang SP (2021). Ecosystem stability and its relationship with biodiversity. Chinese Journal of Plant Ecology, 45, 1127-1139. [李周园, 叶小洲, 王少鹏 (2021). 生态系统稳定性及其与生物多样性的关系. 植 物生态学报, 45, 1127-1139.]

Lindeman RL (1942). The trophic-Dynamic aspect of ecology. Ecology, 23, 399-417.

Ma KP (1993). On the concept of biodiversity. Biodiversity Science, 1, 20-22. [马克平 (1993). 试论生物多样性的概 念. 生物多样性, 1, 20-22.]

May RM (1972). Will a large complex system be stable? Nature, 238, 413-414.

Pan Q, Zheng H, Wang ZH, Wen Z, Yang YZ (2021). Effects of plant functional traits on ecosystem services: a review. Chinese Journal of Plant Ecology, 45, 1140-1153. [潘权, 郑华, 王志恒, 文志, 杨延征 (2021). 植物功能性状对 生态系统服务影响研究进展. 植物生态学报, 45, 11401153.]

Tansley AG (1935). The use and abuse of vegetational concepts and terms. Ecology, 16, 284-307.

Tilman D, Isbell F, Cowles JM (2014). Biodiversity and Ecosystem Functioning. Annual Review of Ecology, Evolution, and Systematics, 45, 471-493.

Violle C, Navas M-L, Vile D, Kazakou E, Fortunel C, Hummel I, Garnier E (2007). Let the concept of trait be functional! Oikos, 116, 882-892.

Wang QQ, Gao Y, Wang R (2021). Review on impacts of global change on food web structure. Chinese Journal of Plant Ecology, 45, 1064-1074. [王晴晴, 高燕, 王嵘 (2021). 全球变化对食物网结构影响机制的研究进展. 植物生 态学报, 45, 1064-1074.]

Wilson EO (1988). Biodiversity. National Academy Press, Washington D.C.

Zhang HJ, Wang W (2021). Responses of ecosystem multifunctionality to global change: progress, problem and prospect. Chinese Journal of Plant Ecology, 45, 1112-1126. [张宏锦, 王娓 (2021). 生态系统多功能性对全球变化 的响应: 进展、问题与展望. 植物生态学报, 45, 11121126.]

Zhong ZW, Li XF, Wang DL (2021). Research progresses of plant-herbivore interactions. Chinese Journal of Plant Ecology, 45, 1036-1048. [钟志伟, 李晓菲, 王德利 (2021). 植物-植食性动物相互关系研究进展. 植物生态 学报, 45, 1036-1048.]

责任编委: 郭 柯 责任编辑: 谢 巍 